\title{
Chapter 5 \\ Pressure: The Bernoulli Principle and Flow Energy Conservation
}

To integrate an airfoil or wing into an airplane one must be able to relate the velocities obtained from an examination of the flow's speed and direction, as we have done above, to the pressures acting on the various parts of the airfoil or wing.

The Bernoulli principle is often given as the reason why wings can provide lift and airplanes can fly. It relates pressure and flow speed ${ }^{1}$ and thus plays an important role in determining aerodynamic forces. The principle states that in a "nice" flow situation, the pressure rises when the medium is slowed and vice versa. The invocation of this relation that we shall state formally as we proceed, is useful because it describes how changes in velocity relate to local pressure. So, where does this principle come from and is it useful for all situations? We will see that it is valid for incompressible flow, i.e., for the description of low-speed air flow or flows of water generally. To cover its application to aviation, we must keep an open mind because airplanes can easily fly in speed regimes where the air is a compressible medium and, it turns out, the compressibility of air has profound influences on the performance of wings and airplanes.

The discussion to follow is limited to air and water. These fluids are involved in situations where the forces for lift and propulsion are commonly of interest, hence the focus of our attention.

The task of relating pressure to the flow conditions is unfortunately not as simple as it may seem. Our approach will be to describe the basic underlying physical law, a law being an observation that has never been seen to be violated. We will explore it in circumstances where the flow is compressible (as it generally is) and apply it in special situations where it may be simplified as incompressible.

The first task is to address the nature of compressible media. This necessarily involves discussion of quantities that describe the state of air, i.e., its properties,

\footnotetext{
${ }^{1}$ The more general form of the energy balance statement, from which the Bernoulli principle is derived, includes accounting for potential energy changes. That feature must be included in a discussion of larger scale flows such as, for example, those associated with weather. For flows associated with flight, potential energy changes play a vanishingly small role so that accounting for them is unnecessary. 
specifically pressure, temperature, density, etc. To the possible chagrin of the reader, this also necessarily involves other air properties that are affected by these parameters. Discussion of such properties involve abstract concepts that may strike fear in the reader. The hope is that it does not. The purpose of this writing is to provide light so that the merit of the conceptual ideas may be appreciated. It should be of some comfort that it took mankind until late in the Industrial Revolution to fully grasp these concepts and utilize them. And did we ever!

\title{
5.1 Air is Compressible (Sometimes)
}

What do we mean by “compressible?" Simply stated, compressibility is related to the fact that pressure changes or temperature changes can lead to fluid density changes. For air, these three quantities are related to each other in a very simple form: a state equation $(p v=R T)$ that many students will have encountered in their science studies. The letters stand for pressure, volume per unit mass, ${ }^{2}$ and temperature. The constant ' $R$ ' is there because we were never smart enough to invent a system of property units to measure $p, T$, and $v$ so that $R=1$. $R$ does carry units that the reader can look up. The temperature and pressure must be stated in values relative to their absolute zeros. Zero temperature is where thermal motion of molecules ceases and zero pressure is called a vacuum. For an incompressible medium like liquid water, a state equation is not particularly interesting because the density (and its inverse, specific volume, $v$ ) is fixed for all practical purposes.

\subsubsection{An Aside: How Good is Air?}

\begin{abstract}
A serious student of the matter will notice that we (aerodynamicists) use the ideal gas description of the gas, namely $p v=R T$. There are circumstances where use of this relationship may have to be revisited. That caution also applies to the other constants that will described in connection with other properties, the specific heats of air in particular. The concern arises when the air's thermal energy depends not only on temperature but also on the pressure. Conditions where our simple descriptions might have to be modified are for situations where the air's oxygen and/or nitrogen are near condensation, i.e., very cold. In such states, gases are described as vapors. Near condensation, intermolecular forces play a role and thus result in a pressure dependence of the internal energy. On the high temperature spectrum end of possibilities, such as conditions reached on vehicles re-entering the earth's atmosphere, air molecules break into atoms and even lose their electrons. The radio silence on re-entry is a manifestation of the ionization ${ }^{3}$ of the air surrounding the vehicle and the resultant inability
\end{abstract}

\footnotetext{
${ }^{2}$ Also termed specific volume and is the inverse of density.

${ }^{3}$ Ionization is the process of breaking down an electrically neutral particle into a positively charged ion and one or more free electrons.
} 
of radio waves to be transmitted through such ionized air. Fortunately for the study of conventional aerodynamics, air, even air with a small amount of water vapor, behaves as a perfect ${ }^{4}$ gas and we can safely ignore all these worries about more complex descriptions of air.

The "nice" flow conditions we consider here are flows along a streamline where speeds may vary. A unit mass of air undergoes a process that will be reflected in the changes in the gas properties. The question has to be: What properties describe the process experienced by a chunk of air as it changes speed? Measures of the energy in the flow and the related temperature and pressure are easily seen to vary. The correct measure of thermal energy is a property of the air and will be identified shortly. Is there another property involved? Perhaps conserved? The answer involves delving into questions about reversibility. In other words, after an increase in speed and a later equal decrease, are the properties describing the air altered or not? Fortunately, under many circumstances of interest to an aerodynamicist, the air that experiences velocity changes by flowing over an airfoil and is returned to the same pressure it had before the airfoil came along is not (measurably) different in density or temperature. It is said to have undergone a reversible set of processes.

The world is full of irreversible processes, but aerodynamic ones of interest to us are fortunately not numerous. They can, however, be challenging. Examples of everyday irreversible processes include the cooling of the cup of coffee on your desk: There can be no realistic hope that the heat that left your coffee will return from the heat in the room to rewarm the coffee. You will have to expend a bit of a valuable resource: the electricity to run the microwave oven. Similarly, all mixing processes are irreversible. Have you ever seen the sugar or cream in your coffee unmix from it without action on your part? Probably not. No sailor stranded at sea has ever been saved by the spontaneous removal of salt from the sea water so he can drink it.

\subsection{Entropy (Arrrgh!@*\#*!)}

A reversible process in air along a streamline conserves a quantity (actually a gas property) called the entropy and the process is termed isentropic (entropy conserving). In much of aerodynamics, flow changes are indeed isentropic. This is really good because high performance requires that as little of the energy that we invest in traveling through air should be left in the environment as a monument to our having done so. In reality, entropy is not conserved and is instead produced, when friction is involved or when irreversible phenomena such as shock waves are experienced. Both of these aspects of flow are of great interest for an aerodynamicist. For that reason, we will consider them in detail. A process is also not isentropic when heat transfer is involved. While the latter aspect is not in play when looking at streamline

\footnotetext{
${ }^{4}$ A gas is thermally perfect when specific heats depend only on temperature. If the specific heat can be assumed constant, the gas is described as calorically perfect. Air, under many circumstances, is perfect in both these senses.
} 
flow over an airfoil, it is very important when the flow through a jet engine combustor is examined. Because flows that do not involve heat transfer are so common in aeronautics, they are given a special name. They are called adiabatic flows. With all that vocabulary in hand, we can summarize our findings with the statement: entropy is conserved when the flow is adiabatic and reversible, otherwise not.

The concept of entropy as a property is a profound one that challenges students in the study of thermodynamics. Understanding this idea, first expressed by the German scientist Rudolph Clausius in the mid nineteenth century, was a scientific tour-deforce whose impact on engineering is hard to overstate. It is usefully applied to the design of engines in particular and answers, in part, questions like: How much of the heat we might supply to an engine can be converted to useful work? This was the central question posed by Sadi Carnot, an officer in Napoleon's army. Part of his genius was recognition of the nature of heat and its convertibility to useful work. His inquiry led to significant progress toward the practicality of steam and other engines in the century and a half that followed his life.

The concept of entropy is very powerful indeed, applying to notions of chemical equilibrium, and even speaks to the cosmic possibility of life, among many other situations. Many, many books have been written about entropy and its significance in connection with the so-called Second Law of Thermodynamics since its formulation late in the nineteenth century. Food for thought is that the Second Law is a disarmingly simple and a seemingly obvious statement: heat cannot flow from a cool place to a hotter one! It is a law because it has never been observed to be violated.

We are concerned (for now) with the narrower problem of adiabatic air flow. The mathematics of entropy conservation can be shown to allow a relationship between the descriptive variables of a gas as it undergoes an isentropic process. For our purposes, let us not delve into such details, save to note that such a description exists and is in fact representative of real processes under many but limited circumstances. The mathematical statement relating air pressure and temperature that is imposed by the isentropic process is quite simple. It says that a changing (absolute) pressure will result in a change in (absolute) temperature raised to the $(\gamma /(\gamma-1)=3.5)$ power. The constant $\gamma(=1.4)^{5}$ is derived from properties of air and is related to the fact that air is primarily composed of the diatomic molecules, oxygen and nitrogen. Its numerical value is highlighted further on. For the interested reader, a mathematical derivation for this relation between pressure and temperature is given in Appendix B.

We return to the question: how do we quantify compressibility? The basis is the understanding that Newton's laws of motion (the various forms and consequences of $F=m a$ ) can be written in differential form, i.e., for small changes in properties and flow conditions. Such a formulation identifies the parameter that quantifies the importance of compressibility in a reversible process. Without going into algebraic details (professors torture students with this and the details are given in Appendix B), one can show that a fractional change in density is always proportional to a fractional change in local speed. The proportionality constant involves the ratio of local speed to the speed of sound, the Mach number, $M$. The constant is $\left(-M^{2}\right)$. To talk about

${ }^{5}$ The parameter $\gamma$ is called the specific heat ratio. 
"low speed" flow, we specifically mean low Mach number flow, say values under $M$ $\sim 0.3$. For example, at this Mach number, a $10 \%$ increase in local speed results in a $\sim 1 \%$ decrease in density, which may indeed be negligibly small, but at the flight speeds near the speed of sound, compressibility effects must be considered for an accurate description of the flow field.

The role of the speed of sound in matters relating to compressibility makes sense because it is the speed with which information spreads from one place to another in the flow field. Thus, wave or information propagation is central to the ability of the flow to adapt (or not) to externally imposed condition changes. Air molecules move with thermal speeds, bounce into each other, and share dynamic information. That implies that the speed of sound in air depends only on the temperature. It does not depend on either the local air pressure or density, nor altitude as such. The temperature in our atmosphere does vary with altitude and with it, there is a corresponding variation in the speed of sound.

Consider some examples with a wide set of descriptive circumstances. Both water and air are fluid media and our review of airfoils is equally valid in air and water. The speed of sound in water is very large, about $1450 \mathrm{~m} / \mathrm{s}$ or over $3200 \mathrm{mph}$. That sound speed is so high that for practical purposes in realistic water flow situations, like the motion of ships, the ratio of flow speed to pressure wave propagation is very small. Water is nearly incompressible because the Mach number-like parameter describing any realistic situation is very, very small. Our concern is primarily with air in which compressibility effects will be significantly more important. Importantly, we note that the sound speed in water is unrelated to the surface wave speed ${ }^{6}$ we describe below in connection with the "hydraulic jump" as an analogy to a shock wave in air. Watercraft dealing with surface waves are involved in a distinct area of study called hydrodynamics where surface waves can be central in importance.

\subsection{Energy Conservation on a Streamline}

So, what about Bernoulli? We have to get back to basics and discuss another concept that a student usually gets exposed to in a course in thermodynamics. We will do here what is necessary in a hopefully in a comprehensible way.

A basic concept involving variables that describe the state (i.e., the pressure, temperature, etc.) of the air as it undergoes velocity changes is an energy conservation statement, or what is formally termed an energy equation. In a moving fluid there are two forms of energy (per unit mass) when we justifiably neglect changes in potential energy. The first is kinetic energy $\left(1 / 2 V^{2}\right)$ and the other is thermal energy; here $V$ is the speed of the air.

The thermal energy is the (randomized) kinetic energy of the atoms or molecules of the air and is measured by the (absolute) air temperature. The absolute temperature

\footnotetext{
${ }^{6}$ The wave speed in water depends on the depth of the water. For example, in a pool of water 1 (10) foot $[30 \mathrm{~cm}(3 \mathrm{~m})]$ deep, the wave speed is about $5.6(17.9) \mathrm{feet} / \mathrm{s}$ or $\sim 2(3) \mathrm{m} / \mathrm{s}$.
} 
scale is not in common use so that it must be determined from knowledge of absolute zero temperature. On a Celsius or Centigrade scale, water ice (at one atmosphere pressure) freezes at zero degrees $\mathrm{C}$ or $273 \mathrm{~K}$ (degrees Kelvin) which corresponds to 32 F or 492 R (degrees Rankine) in the English system. The Kelvin and Rankine temperatures are absolute scales. The thermal energy associated with molecular motion is formally called the internal energy of the fluid and typically described by the algebraic symbol, $u$.

\subsection{Enthalpy is a Property. Really? Really!}

The introduction of the word 'energy' in connection with random motion of molecules is an opportunity to digress to explore the nature of heat and energy. Formally, the energy conservation statement is called the First Law of Thermodynamics. Its formulation does two things: it identifies specifically the energy of a substance as a property and defines the nature of heat. The nature of heat puzzled the early investigators to a great degree. The First Law of Thermodynamics allows us (modern engineers and scientists) to state clearly that heat is not a property, but it is that which travels from a hot body to a colder one. This may sound like a mind-game, but it is part of a necessary construct that allows understanding of the nature of heat, work and all that follows from exploitation of that understanding. This is just the topic that Carnot wrestled with. One might wonder just what is a hot or colder body? That, to a modern mind, involves the concept of temperature, that is laid down as the Zeroth Law of Thermodynamics: there is such a thing as temperature! The concept of temperature and its connection with heat was not nearly as well understood more than a century ago as it is today. Consider, for example, the confusion contributed by the reality of phase changes such as melting ice or condensing steam: heat is involved without temperature changes. The zeroth law also points to the existence of the absolute zero in temperature. But enough of the digression into theoretical worlds and let's return to the real one.

The matter of describing the energy equation (or First Law) for a moving fluid is somewhat complicated by the fact that adjacent fluid elements do work on one another as velocity changes are imposed. Picture, for example, the compression of air by a bicycle pump. The air in the pump space is compressed, that is, work is put into the confined air (with a force that reduces the volume) and that results in an increase in temperature. In an antiparallel way, carbon dioxide gas in a fire extinguisher does work to push out what leaves the cylinder when you pull the trigger and that results in a decrease in the temperature of the gas remaining in the cannister. In a flow situation, the work is put into (or removed from) any (and all) elements, not by a piston, but by the pressure forces from its neighbors. Such work interactions affect the state of the gas, specifically its internal energy. An accurate energy conservation statement must account for this work. Work exerted in this way is called flow work and is described by pressure times volume ( $p v$, the thermodynamic equivalent of mechanical force 
times distance). This term is per unit mass because the quantity ' $v$ ' is the volume per unit mass.

Making life easier for aerodynamicists, the product " $p v$ " is also linearly related to the temperature through the state description of air cited above: the ideal gas law. The energy conservation statement that applies to a moving fluid is therefore not just about the internal energy $(u)$ but a new quantity consisting of that energy and flow work. This quantity is the enthalpy ${ }^{7}$ (symbol $h$, defined as $u+p v$ ) and, fortunately for air, it is also proportional to just the temperature. It is this measure of thermal energy content in the element that is traded with the flow kinetic energy in our ordinary stream tube. If one defines something called the total enthalpy (symbol $h_{\text {tot }}$ ) of the flow as the sum of static (proportional to temperature) enthalpy and the kinetic energy, then it is the total enthalpy that is conserved in a streamline-like (adiabatic) flow. With a total enthalpy thus defined, the notion of total temperature, namely, the temperature that the fluid would have when it is brought to rest, stumbles right out. In algebraic form, the energy conservation statement for our circumstances is

$$
h+1 / 2 V^{2}=h_{t o t} \text { is conserved. }
$$

The concept of total temperature is a very useful tool to describe the state of a moving gas along a streamline. The understanding to this point allows that quantity to be written in terms of velocity but, much more convenient is writing it in terms of Mach number. That speed ratio characterizes the velocity in terms of the other relevant speed that is a property of a compressible medium. For the understanding of the motion of compressible media, such as air, it is a requirement to adopt the view that speed is better expressed as Mach number, rather than speed itself. The mathematical and physical descriptions would be excessively complicated. This switch to Mach number as the important flow speed parameter was a key breakthrough by the early aerodynamicists in the 1920 s.

An important and practical consequence of the conservation of total enthalpy and temperature is the idea of a total pressure. For an isentropic process, the conservation of total enthalpy allows the definition of a pressure resulting from bringing the flow to rest in a gentle, reversible way. The usefulness of that total pressure is in its ability to describe the irreversible (adiabatic, no heat supply or removal) losses associated with a process such a compression in a compressor or flow through a shock wave. Thus, there are total pressure reductions involved in irreversible processes like friction, heat addition, etc., but for a reversible (adiabatic) process total pressure is conserved together with the total temperature.

\footnotetext{
${ }^{7}$ Enthalpy (common symbol $h$ ) is the sum of (internal energy, $u$, that depends only on temperature) and flow work $(p v)$. For air, the enthalpy is thus directly proportional to the temperature because air is an ideal gas $(p v=R T)$.
} 


\subsubsection{A Few Details About Specific Heats}

This paragraph can be skipped but may be helpful. The intent is to shed light on the origin and nature of the quantity, ' $\gamma$ ', introduced earlier. The internal energy (per unit mass) is proportional to the absolute temperature as is the enthalpy. The proportionality constants for these are called specific heats. These quantities are the amount of heat required to raise the temperature of a unit mass of air by one degree. For the internal energy, one can imagine an experiment where one heats the gas in a rigid container to obtain the so-called specific heat at constant volume, symbolized by $C_{v}$. One can do the same experiment at constant pressure by allowing the test air sample to raise a piston (that keeps the pressure constant) as it is heated. The heat required will be greater because, in addition to raising the temperature of the sample, work is done with a force moving the constraining boundary (force, the weight of the piston, times distance upward). The resulting quantity is the specific heat at constant pressure, $C_{p}$, which is always greater than $C_{v}$. From their definitions and that of the enthalpy, $h$, the difference is the ideal gas constant, $R$.

For our purposes, both of these specific heats can be taken as constants for typical aerodynamic problems with air. Their numerical values depend on the system of units used (English or metric). Their ratio is, however, dimensionless. In air, the ratio of these two specific heats comes up so often that it is given its own symbol, $\gamma$, and is numerically equal to 1.4 for air under normal circumstances of interest to flight. In algebraic relations, the combinations $(\gamma-1) / 2$ and $\gamma /(\gamma-1)$ appear frequently. Numerically, they are 0.2 and 3.5 respectively but we will keep them in the general form because, well, it looks cool!

So, what have we accomplished so far? We have introduced two abstract quantities that are useful in the description of a gas as it proceeds along a streamline. Looking at the relatively simple flow situation along a streamline, we find that the sum of static enthalpy and kinetic energy are conserved and temperature is an excellent measure of the enthalpy. Thus, as velocity increases in the flow over the top of a wing or an airfoil, the (static) temperature measured by a mosquito in that flow element will fall and his environment will warm up again when the flow slows down, while the total enthalpy as characterized by a total temperature remains unchanging.

The second concept is that of the entropy and its close connection to process reversibility. While it cannot be measured by an instrument (nor purchased by the pound), its practical connection is to the total pressure that can be measured. Appendix B sets out a quantitative description of entropy and its relation to measurable descriptive gas properties.

\subsection{Total Temperature}

The definition of total temperature that is often used to describe the heat and work interactions and will be addressed further on in connection with the jet engine. Thus, rewriting the enthalpy conservation statement above in terms of temperature and Mach number, we have:

$$
T_{\text {tot }}=T\left(1+\frac{\gamma-1}{2} M^{2}\right)
$$


For air, the speed of sound, $a$, is given by

$$
a=\sqrt{\gamma R T} \text { and Mach number, } M=V / a
$$

Mach number is a good way to characterize flow speed when dealing with flows that are even a little bit compressible. This statement will become more evident when we talk about the pressure variation along the streamline and the failure of the Bernoulli statement to yield accurate pressures when applied to compressible flows (see discussion at the end of this chapter).

When entropy is conserved, the pressure varies relatively simply with the temperature. As temperature increases so does the pressure and vice versa. This was the last link we needed to be able to relate flow velocity changes to pressure on airfoils and from that deduce forces of interest for flight. The details will be revealed a little further on but, first, a look at a practical manifestation.

To illustrate that the relationship between air temperature and speed we can look at a wing generating lift where we know that the flow proceeds over the wing at high speed. The discussion above suggests that the temperature in the region above the wing should be lower than ambient. If the ambient air is high in humidity, then a decrease in temperature will result in water condensation. Figure 5.1 shows the phenomenon. One might look at this and say that the flow can't be adiabatic because

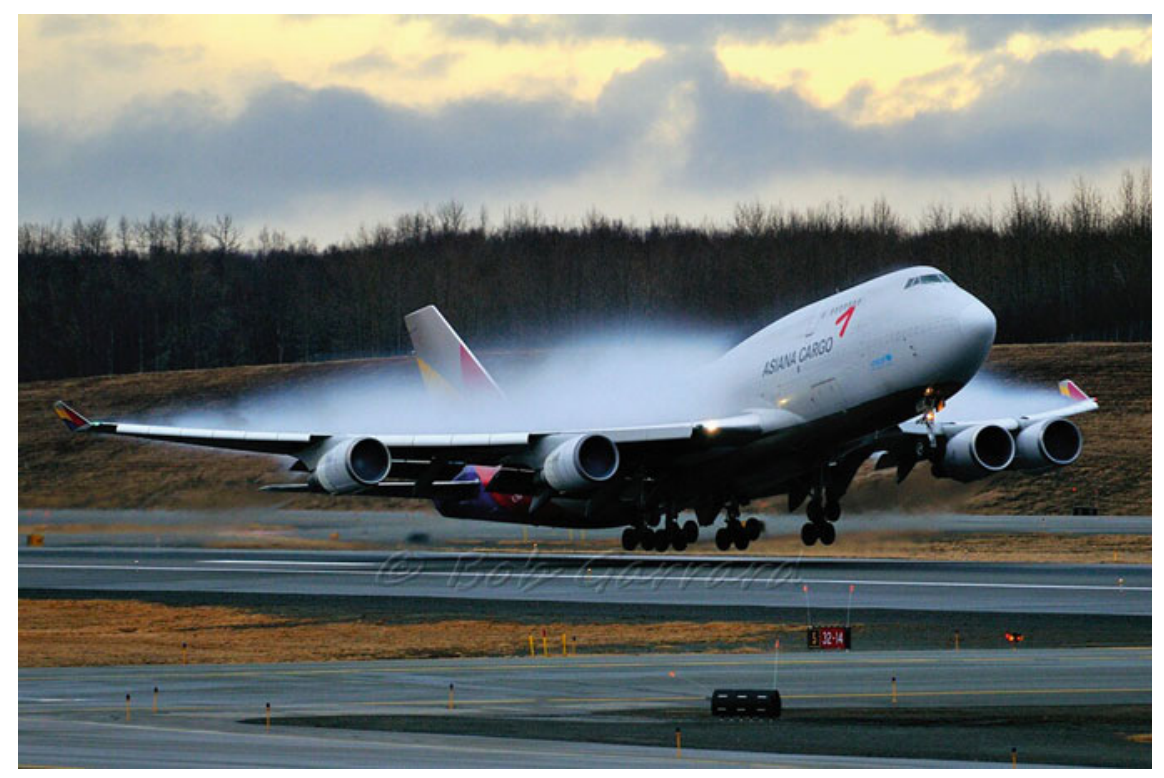

Fig. 5.1 A Boeing 747 at takeoff on a humid day with condensation cloud above the wing (Photo courtesy Bob Garrard) 
condensation is taking place. After all, there is heat involved in condensation. Fortunately, the amount of heat relative to the thermal energy in the air is quite small and we can safely view the flow as close to adiabatic.

With the isentropic relationship between temperature and pressure, the cloud can be viewed as something of a representation of the pressure or lift distribution on the wing. Similar condensation phenomena can sometimes also be seen at the tip vortex of propeller and helicopter rotor blades.

\subsection{Bernoulli at Last}

We have all the tools required for determining pressures and forces on a wing or airfoil. It may, nevertheless, be convenient to step back and look at the Bernoulli principle because that was the tool early investigators had to analyze aerodynamic issues. Further, the Bernoulli form of the First Law makes for an easier understanding of flow phenomena ... provided the assumption of incompressible fluid is acceptable.

With that limitation in mind, the conservation of total enthalpy for flows of moving water (or low Mach number air) is much simpler. The balance is not between temperature and speed because work, specifically flow work, cannot be done on the fluid because it is incompressible: it cannot change volume. Hence the temperature (as a measure of internal energy) is unchanged as speed changes occur along a streamline. The result is that for incompressible fluids, only the pressure in the flow work term adjusts to speed changes. The energy balance statement that arises is the Bernoulli principle and applies strictly to incompressible fluids like water. For all practical purposes, the enthalpy is a function of only the pressure in this case. The relation between pressure $(p)$ and speed $(V)$ for an incompressible fluid is therefore the familiar statement

$$
p v+1 / 2 V^{2} \text { or } p+1 / 2 \rho V^{2} \text { are conserved along a streamline (the density } \rho=1 / v \text { ) }
$$

In short, as $V$ increases, $p$ must decrease. Further, the forces on a wing consequently vary as the square of the speed.

The failure of this relation to be accurate rests with the fact that, for compressible flows, both pressure and density vary. Therein lies the usefulness of Mach number to characterize compressible flow speeds, rather than using the speed itself. Although it is not as commonly expressed that way, the Bernoulli statement can be expressed (for air) in terms of Mach number as

$$
p\left(1+\frac{\gamma}{2} M^{2}\right)=p_{t o t} \text { is conserved (incompressible flow) }
$$

For the more general statement applied to compressible air, the mathematical statement is somewhat more complicated ${ }^{8}$

8 ... but amounts to the same expression as above when $\mathrm{M}$ is small. 


$$
p\left(1+\frac{\gamma-1}{2} M^{2}\right)^{\gamma / \gamma-1}=p_{t o t}
$$

\subsection{Total Pressure}

Note in both statements, the notion of total pressure is invoked. The total pressure is sometimes referred to as the stagnation pressure, the pressure realized by bringing the flow to rest, nicely. The latter statement follows directly from the definition of total temperature and the relationship between pressure and temperature for an isentropic process.

To put the matter of Bernoulli as an approximation to rest, we note the pressure at the stagnation point of a streamline, namely the pressure realized when the flow comes to rest in air is as shown in Table 5.1 and calculated from the ratio $\mathrm{p}_{\text {tot }} / \mathrm{p}$ for a given Mach number.

The two values are in fair agreement at $\mathrm{M} \sim 0.3$ but differ to a substantial degree at higher Mach numbers.

In closing, one can say that the Bernoulli relationship can be used for a qualitative description of the effect of local speed changes on a surface as they impact the local pressure.

Table 5.1 The pressure rise from 1.0 for bringing a flow to rest from a flow at the Mach number noted

\begin{tabular}{l|l|l|l}
\hline Mach no & 0.3 & 0.8 & 1 \\
\hline Bernoulli & 1.061 & 1.43 & 1.68 \\
\hline True value & 1.064 & 1.52 & 1.89 \\
\hline
\end{tabular}

The results are given by the Bernoulli equation as well as by the correct calculation using the energy equation. For $\mathrm{M}>1$ shock waves are involves and flow description involves more complex aerothermodynamics and using Bernoulli is certainly not a correct approach 
Open Access This chapter is licensed under the terms of the Creative Commons Attribution 4.0 International License (http://creativecommons.org/licenses/by/4.0/), which permits use, sharing, adaptation, distribution and reproduction in any medium or format, as long as you give appropriate credit to the original author(s) and the source, provide a link to the Creative Commons license and indicate if changes were made.

The images or other third party material in this chapter are included in the chapter's Creative Commons license, unless indicated otherwise in a credit line to the material. If material is not included in the chapter's Creative Commons license and your intended use is not permitted by statutory regulation or exceeds the permitted use, you will need to obtain permission directly from the copyright holder.

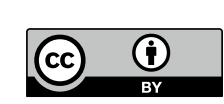

